\title{
A MODIFIED TWO DIMENSIONAL ADAPTIVE DIGITAL FILTER
}

\author{
M. Fouad ${ }^{1}$, A. F. Abdel-Razek ${ }^{2}$ and H. M. E. Hamada ${ }^{2}$ \\ ${ }^{1}$ Electronics and Communications Dept., Zagazig University \\ ${ }^{2}$ Electrical and Computer Engineering, Higher Technological Institute, \\ Tenth of Ramadan
}

\begin{abstract}
In this paper, a new scheme of recursive two-dimensional (2D) adaptive digital filter is presented. It consists of a forward section, a feedback section, and decision block. A little complexity increase from [10] is used by adding adaptive quantizer to predictor error. Each section is realized as conventional non-recursive adaptive filter. Either 2D adaptive LMS or sign function algorithm can be used for adaptation. The proposed recursive filter operates as a $2 \mathrm{D}$ adaptive line enhancer (ALE) for images contaminated with noise. Its performance is compared with that given in [1].
\end{abstract}

\section{KEYWORDS : Recursive Two-Dimensional (R-2D), Adaptive Line Enhancer (ALE)}

\section{INTRODUCTION}

The subject of adaptive filtering has established itself as an important branch of statistical signal processing, with applications in various interesting fields particularly in image. The two dimensional signal can be modeled as nonstationary $2 \mathrm{D}$ random field in which the filter design is based. Thus, the resultant filter is no longer optimum. This necessitates the use of 2D adaptive filters.

Indeed, the adaptive filtering problem is to find an algorithm for adjusting the filter parameters in a situation where complete knowledge of the relevant signal characteristics are not available, so that the performance of the adaptive filter may convergent to that of the optimum filter after a sufficiently large number of iterations of the algorithm. The pioneering work of Hadhoud Thonous [1] extended the 1D least mean square (LMS) adaptive 
algorithms (Widrow's algorithm [2] to the 2D case. In [3] Jenkins and Faust proposed a similar algorithm, joined to the McClellan transformation. Although these algorithms can successfully get the filter coefficients, they update the filter confidents only along the horizontal direction of the 2D plan. Consequently, these LMS algorithms are inherently 1D adaptive algorithm which doesn't fully utilize the information content of the 2D signals besides the performance degradation due to an isotropic processing.

Usually adaptive digital filters are classified into non-recursive or finite impulse response (FIR) filters, and recursive or infinite response (IIR) filters. Non-recursive digital filters are inherently stable, but require two times as many coefficients as recursive filters when compared to same performance index. Recursive adaptive filters may suffer from two major problems due to feedback: a) they may be unstable, and b) the adaptation of their coefficients may drive same poles outside the unit circle. Thus, recursive adaptive filters are usually susceptible to instability. Despite these difficulties, adaptive recursive filters are preferred. Particularly when processing a large amount of 2D data, because they are less complex and more fasted adaptable than non-recursive filters.

In this paper, a modified model of 2D adaptive recursive digital filter is presented. It consists of two non-recursive parts: the forward section and feedback section. The filter coefficient are adjusted in accordance with the 2D least mean square method (2D LMS) and the 2D-sign-algorithm which converges to both the horizontal and the vertical directions of the $2 \mathrm{D}$ plane. A scalar four level, adaptive quantizer is used to quantize the prediction error. To evaluate the performance of the proposed scheme, the adaptive recursive filter is implemented as a 2D adaptive line enhancer (ALE) of an image contaminated with noise. The performance the ALE presented is compared with that of the ALE presented by Hadhoud in [1]. The proposed ALE proves its superiority in noise reduction when applied to noisy images.

\section{THE PROPOSED MODEL OF THE 2D ADAPTIVE RECURSIVE DIGITAL FILTER}

Given the $2 \mathrm{D}$ recursive filter transfer function; $\mathrm{H}\left[z_{1}, z_{2}\right]$ of the form

$$
\mathrm{H}\left[z_{1}, z_{2}\right]=\mathrm{B}\left[z_{1}, z_{2}\right] / \mathrm{A}\left[z_{1}, z_{2}\right]
$$

and writing the denominator polynomial as follows

$$
\mathrm{A}\left[z_{1}, z_{2}\right]=\mathrm{k}+\mathrm{C}\left[z_{1}, z_{2}\right]
$$

Where the polynomial $\mathrm{C}\left[z_{1}, z_{2}\right]$ does not contain any constant term ( see [4] ), then the filter transfer function will be

$$
\mathrm{H}\left[z_{1}, z_{2}\right]=\mathrm{B}\left[z_{1}, z_{2}\right] /\left(\mathrm{k}+\mathrm{C}\left[z_{1}, z_{2}\right]\right)
$$

$\mathrm{H}\left[{ }^{z_{1}}, z_{2}\right]$ can be realized by a forward section, $\mathrm{B}\left[{ }^{z_{1}}, z_{2}\right] / \mathrm{k}$, and a feedback section, $\mathrm{C}\left[{ }_{1}, z_{2}\right] / \mathrm{k}$. We exploit this property in the design of the proposed 2D adaptive recursive filter depicted in figure 1 . Both the forward and feedback sections are non-recursive adaptive digital filters which can be realized using conventional method. 
The restriction on the actual implementation of the 2D adaptive digital filter of figure 1 is reliability. Unrealizable delay-free loops in the structure must be avoided and this can be accomplished by graph transformation of the resulting filter [5]. The purpose of the decision block shown in figure 1 is to avoid instability which might arise from adaptation of the feedback section coefficient. Stability is ensured due to the boundedness of the feedback signal.

\subsection{Filter Adaptation}

Regardless the filter type, the filter coefficient should reach their optimum values using an adjustment method (adaptation algorithm) which involves updating each coefficient during each sequence sample. The importance of a specific algorithm is to be underlined as it will directly influence both the transient and the steady state performance of the filter as well as complexity of its implementation. In most applications, the least mean square algorithm is recommended because of the easy implementation and the favorable maladjustment versus convergence speed trade off. In our model, we use the 2D LMS algorithm for adaptation of both forward and feedback sections.

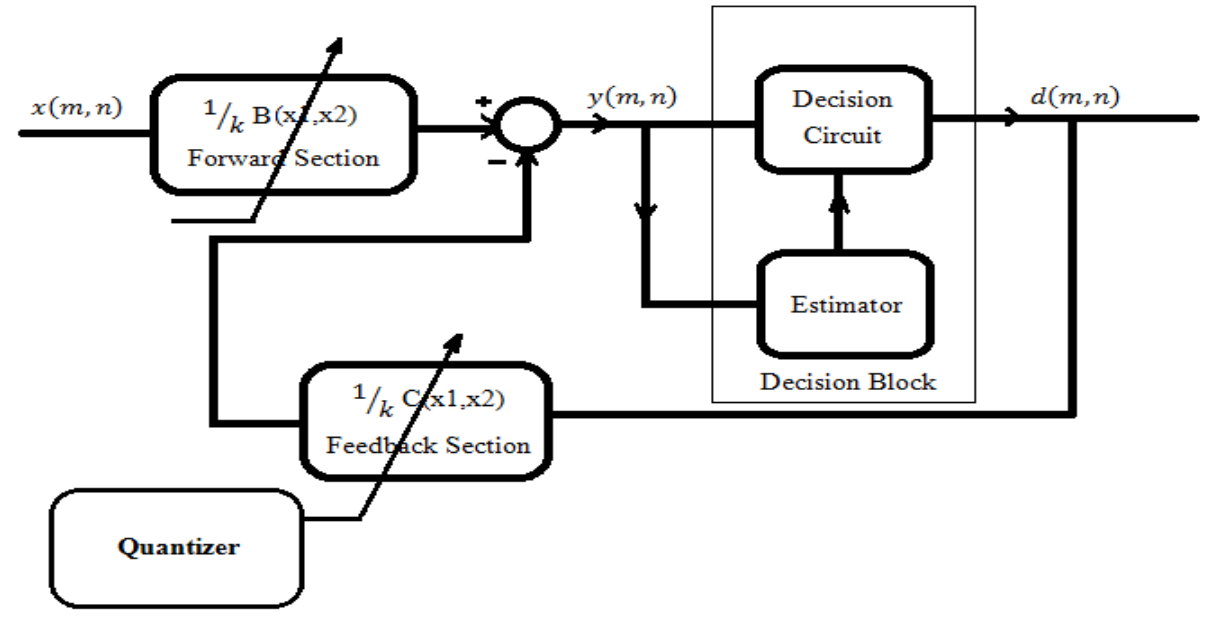

Figure (1): The proposed 2D adaptive recursive digital filter

As stated in the previous section, each part of the proposed 2D adaptive recursive digital filter is a non-recursive filter, represented by the following convolution:

$y(m, n)=\sum_{k=0}^{m-1} \sum_{l=0}^{n-1} \boldsymbol{h}(k, l) x(m-k, n-l)$

Where $y(m, n)$ and $x(m, n)$ are the output and the reference 2D input signals respectively. The support of the $2 \mathrm{D}$ signal is assumed to be of size $\mathrm{M} \times \mathrm{M}$. The filter coefficient are $\boldsymbol{h}(k, l)$ and of $\mathrm{N} \mathrm{x} \mathrm{N}$ support. B 
In order that the output $y(m, n)$ tracks the desired input, the 2D LMS algorithm updates the filter coefficients are $\boldsymbol{h}(k, l)$, via the following equation (5):

$\boldsymbol{h}_{m+1, n+1}(k, l)=f_{\boldsymbol{h}} \boldsymbol{h}_{m, n+1}(k, l)_{+} f_{v} \boldsymbol{h}_{m+1, n}(k, l)_{+}$ $\mu_{\boldsymbol{h}} e(m, n+1) x(m-k, n-l+1)_{+}$

$\mu_{v} e(m+1, n) x(m-k+1, n-1)$

With the initial values $\boldsymbol{h}(k, l)=0, m=0, \ldots \ldots \ldots$ a and $n=0, \ldots \ldots \ldots \mathrm{N}$, where $\boldsymbol{h}_{m+1, n+1}(k, l)$ are the filter coefficients at the number $(m+1, n+1)$ an.d $f_{\boldsymbol{h}}, f_{v}$ are the positive scaling factors satisfying the identity $f_{\boldsymbol{h}}+f_{v}=1$. $\mu_{\boldsymbol{h}}, \mu_{v}$ are step sizes in horizontal and vertical directions, respectively and $e(m, n)$ is the error between the filter's output and desired signal.

\section{$d(m, n)$}

$$
e(m, n)=
$$

The filter used in (4) is an $\mathrm{N}^{\times} \mathrm{N}$ casual filter

$$
x(m, n)
$$

When the reference signal, , and the desired signal, $d(m, n)$, are stationary, the algorithm in equation (5) converges into mean square sense to an optimal solution as $m+n \rightarrow \infty$. The optimum coefficient vector $\mathbf{H}_{\text {opt }}$ satisfies the 2D Weiner-Hopf equation given by:

$$
\mathrm{R}^{\mathbf{H}_{\text {opt }}=\mathrm{P}}
$$

Where the covariance matrix $\mathrm{R}$ is defined:

and

$$
R=\left[\begin{array}{clll}
R_{o} & R_{1} & \cdots & R_{1-N} \\
R_{o} & R_{1} & \cdots & R_{2-N} \\
R_{N-1} & R_{N-2} & \cdots & R_{o}
\end{array}\right],
$$

$$
R_{\mathbf{1}}=\left[\begin{array}{cccc}
r_{1, o} & r_{1,-1} & \cdots & r_{1,1-N} \\
r_{1,1} & r_{1,0} & \cdots & r_{1,2-N} \\
r_{1, N-1} & r_{1, N-2} & \cdots & r_{1,0}
\end{array}\right]
$$




$$
r(k, l)=E\{x(m, n) x(m-k, n-l)\},
$$

$\mathrm{E}\{$.$\} is expectation operator.$

The matrix $\mathrm{R}$ is real, symmetric, or blocks Toeplitz and generally non negative definite. It is here assumed positive definite. The optimum coefficient vector $\mathbf{H}_{\text {opt }}$ is defined by:

$\mathbf{H}_{\text {opt }}=\left[\begin{array}{rllc}\mathbf{H}_{\text {opt }}(\mathbf{0}, \mathbf{0}) & \mathbf{H}_{\text {opt }}(\mathbf{0 , 1}) & \cdots & \mathbf{H}_{\text {opt }}(\mathbf{0}, N-\mathbf{1}) \\ \mathbf{H}_{\text {opt }}(\mathbf{1}, \mathbf{0}) & \mathbf{H}_{\text {opt }}(\mathbf{1}, \mathbf{1}) & \cdots & \mathbf{H}_{\text {opt }}(\mathbf{1}, N-\mathbf{1}) \\ \mathbf{H}_{\text {opt }}(N-\mathbf{1}, \mathbf{0}) & \mathbf{H}_{\text {opt }}(N-\mathbf{1}, \mathbf{1}) & \cdots & \mathbf{H}_{\text {opt }}(N-\mathbf{1}, N-\mathbf{1})\end{array}\right]$

The vector $\mathrm{P}$ is the cross correlation vector between $d(m, n)$ and $x(m, n)$ given by:

\section{$P(0,0)$}

$\mathrm{P}=[$

$$
{ }_{, P} P(k, l)=\mathrm{E}\{d(m, n) x(m-k, n-l)\}
$$

The condition of the convergence of the 2D LMS algorithm given in [6, eq.(5)] is:

$$
\left|f_{\boldsymbol{h}}-\mu_{\boldsymbol{h}} \lambda_{i} \mathrm{I}+\right| \mathbf{f} f_{v}-\mu_{o} \lambda_{i} \mid<1, \quad 1 \leq i \leq N,
$$

Where is an Eigen value of the matrix R

Assuming $f_{\boldsymbol{h}}=f_{v}=0.5$ and that $\mu_{o}=\mu_{\boldsymbol{h}}$ reduces to:

$$
[1-\mu \lambda \mathrm{I}<1, \quad 1 \leq i \leq N,
$$

Which implies that $\lambda \neq 0,1<i<N$, i.e. the matrix $\mathrm{R}$ must be positive definite.

Furthermore the constant $\lambda$ should satisfy the set of equalities (10): 


$$
0<\frac{2}{\lambda_{\max } \bar{\Xi}}
$$

Where implies that $\lambda_{\max }$ is the largest Eigen value of R. From (11), we find that the condition of the convergence of 2D LMS algorithm, is the same as that of 1D LMS algorithm.

As in the 1D case [7], the positive parameter $\mu$ controls the convergence of the 2D LMS algorithm. Convergence is ensured by choosing $\mu$, according to equation (11). As $\mu$ increases, both the convergence speed and the mean square error increase and vice versa. Thus, the choice of $\mu$ is a compromise between the speed and accuracy of the convergence.

One may ensure both rapid and convergence through the use of a large value of, at the start of the adaptation and a smaller one after convergence has been approached. In practical limited precision digital implementation of adaptive filter, it is not advisable to choose a very small value of $\mu$. Indeed, if a very small value of $\mu$ is used; the coefficient correction terms in the algorithm given by equation (5) may become smaller than half of the least significant bit of the coefficient. Thus the adaptation stops and the mean square error remains the same.

As a result of using the 2D LMS method, the filter coefficients do not exactly reach their optimum values and in the steady state the mean square error (MSE) of the output is larger than that obtained when using optimum coefficients.

The MSE is calculated as:

$$
M S E=\frac{1}{(M-N)^{2}} \sum_{m=N}^{M-1} \sum_{n=N}^{M-1} e(m, n)^{2}
$$

To simplify the implementation of the 2D LMS algorithm without seriously affecting its performance, a simple update scheme using non-linear multipliers to produce the coefficient correction terms is introduced to reduce the implementation complexity at the cost of performance. In such scheme, the sign (clipped) algorithm [8] is used. It is given by equation (13):

$$
\begin{aligned}
& \boldsymbol{h}_{m+1, n+1}(k, l)=f_{\boldsymbol{h}} \boldsymbol{h}_{m, n+1}(k, l)_{+} f_{v} \boldsymbol{h}_{m+1, n}(k, l)_{+} \\
& \mu_{\boldsymbol{h}} e(m, n+\mathbf{1}) \operatorname{sgn}[x(m-k, n-l+\mathbf{1})]_{+}
\end{aligned}
$$

Where $\operatorname{sgn}(x)$ is defined as: 


$$
\operatorname{sgn}= \begin{cases}+1, & \text { if } x>0, \\ -1, & \text { if } x \leq 0,\end{cases}
$$

In this algorithm, the signal used for updating the coefficients is corrupted by a great amount of quantization noise. Consequently, the step sizes, and used with the sign algorithm should be smaller than those used with 2D LMS algorithm in order to maintain the same convergence accuracy. Hence, if the speed of convergence is not a problem, the sign algorithm may be very attractive for adaptive processing due to extreme simplicity of its implementation.

\subsection{Adaptive Quantizer}

A scalar four-level adaptation is used to quantize the prediction error. A normalization factor $\delta$ is used in order to consider the dispersion of the prediction error. The normalization factor is adapted by a backwards technique [3]. The correction of the quantizer parameter uses the quantized value of the prediction error $\varepsilon$.

The normalization factor is updated by the following algorithm [3]:

$$
\begin{gathered}
\delta(i, j)=\frac{\sigma(i, j-1) \sigma(i-1, j)}{\sigma(i-1, j-1)} \\
\varepsilon_{1}(i, j)=Q\left(\frac{\varepsilon(i, j)}{\delta(i, j)}\right) \\
\bar{\varepsilon}(i, j)=\varepsilon_{1}(i, j) \delta(i, j) \\
\sigma(i, j)=M\left(\varepsilon_{1}, i, j\right) \delta(i, j)
\end{gathered}
$$

Where, $M(\varepsilon, i, j)$ is a positive value function which for the four-level quantizer satisfies:

$$
M(q)=M(-q)>1 ; \quad M(0)<1
$$

\subsection{Decision Block}

The decision block is a conventional decision circuit with multiple threshold levels. For example, in case when the data sequence takes one of the $2 \mathrm{M}$ levels:

$$
(\mp 1, \mp 3, \mp 5, \ldots ., \mp(2 M-1))
$$

The decision circuit will have (2M-1) threshold levels, their values will be:

$$
0, \bar{\mp} 2 L, \bar{\mp} 4 L, \ldots \ldots, 2(M-1) L
$$

In order to determine the threshold levels, the value of $\mathrm{L}$ must be estimated. The method we use for estimation is as follows: 
In digital systems, the signal to noise ratio (SNR) is usually high and then:

$$
E\{y(m, n)\}=|\mathbf{L}| E\{d(m, n)\}
$$

Since $E\{d(m, n)\}$ is known quantity, given by the statistical characteristics of the input data $\mathrm{L}$, can be obtained by a time averaging circuit applied to $y(m, n)$ and whose gain is adjusted to the value $1 / E\{L\}$, the noise will affect the estimate of $\mathrm{L}$.

For high SNR, this may be ignored. The value of $|L|$ is used instead of $L$ in assigning the values of the threshold levels. In case when $\mathrm{L}$ is positive, this makes no difference. However, when $L$ is negative then using $|L|$ instead of $L$ will result in sign inversion of the whole data sequence. In most practical cases [9], sign inversion of the whole data sequences is allowed. If this is not the case, then an easy measure that depends on the protocol of data processing can recognize and correct this sign inversion.

\section{2D Adaptive Line Enhancer}

Here, we consider the 2D adaptive line enhancer (ALE) as an application to the proposed $2 \mathrm{D}$ adaptive recursive digital filter described in the previous section. The 2D ALE which depicted in figure 2 is an adaptive filter for noise reduction.

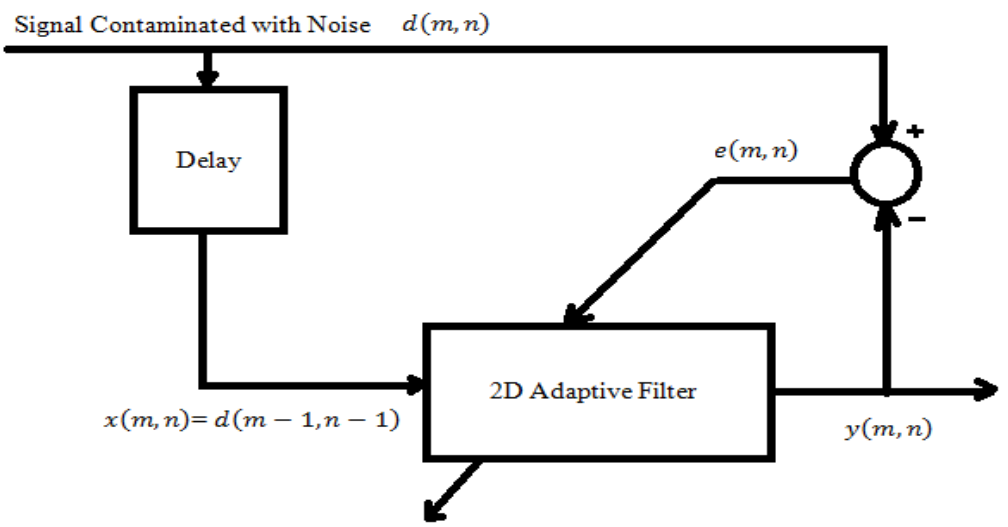

Fig. (2): The 2D adaptive Line Enhancer

As shown in figure 2, the desired input $d(m, n)$ is the input signal and the

$$
d(m, n)
$$

reference signal $x(m, n)$ is the delayed version of . The signal $y(m, n)$ represents the output of the ALE, the ALE implemented and its performance is examined. The used input signal is an image of size $128 \times 128$ and the filter mask size is $3 \times 3$ i.e. $\mathrm{M}=128$ and $\mathrm{N}=3$. The images of a girl 
(128 x 128) produced by the applied Mathematics and Computing Group laboratory of Cranfield institute of Technology is used in our experiments. The ALE based on the proposed 2D adaptive digital filter is designed using 2D LMS algorithm with $f_{\boldsymbol{h}}=f_{v}=0.5$ and $\mu_{\boldsymbol{h}}=\mu_{v}=\mu$ and we call it ALE 1 . For the proposal of comparison the ALE based on Handhold's 2D LMS algorithm [1] is also implemented and called ALE 2. In both ALE's the value of parameter $\mu$ is chosen to be the optimum value that makes the mean square error (MSE) between the desired input and the output minimum and it is decided by try and error.

To evaluate and compare the performance of ALE based on the proposed adaptive filter, first the ideal image of figure 3 is applied to ALE 1 and ALE 2. The values taken by $\mu$ and MSE are $2.1 \times 10^{-6}$ and 225 respectively, while the ALE 2 they take the values $\mu=1.6 \times 10^{-6}$ and MSE $=169$. The results are shown in figure 4 and 5. The image processed by the ALE's is blurred to some extent. The image processed by the ALE 1 is sharper than that of the ALE 2. One can find that the MSE of the ALE 2 is smaller than that of the ALE 1. That is because the traceability of ALE 1 to local static of images whose statistics vary gradually is superior to that of the ALE 2. The blur caused by ALE 1 is greater than that of ALE 2 .

In second experiment, the performance of the ALE's is evaluated and compared in the presence of noise. The ALE 1 with $=1.9 \times 10^{-6}$ and MSE $=$ 341 , and the ALE 2 with $\mu=6.5 \times \mathbf{1 0}^{-7}$ and MSE $=325$ are designed. The noise image of figure 6 is applied to the two ALE's. The noise is additive Gaussian white noise with zero mean and its variance is adjusted to make the signal to noise ratio (SNR) 6dB. The results showed in figures 7 and 8 . The ALE1 produces better results of noise reduction in flat areas than that of ALE 2 , and the effect of ALE 2 for noise reduction in edge areas is almost equal to ALE 1. The ALE 1 does not impose any degradation as in the case of ALE 2. Finally, the ALE is implemented using the 2D sign algorithm with $f_{h}=f_{v}$ $=0.5$ and $\mu_{\boldsymbol{h}}=\mu_{v}=\mu$ and compared with ALE 1 . Again, the parameter $\mu$ is chosen by try and error to obtain the minimum MSE. $\mu$ takes the value $1.5 \mathrm{x}$ $10^{-7}$ and MSE $=192$. It is noticed that sign algorithm converges more slowly than the 2D LMS algorithm.

However, when $\mu$ are quite small both algorithms ensure almost the same MSE. It should be reminded that the main advantage of the sign algorithm is its simple implementation. Consequently, the sign algorithm is recommended for the cases, in which the interest is the technical simplicity rather than the convergence speed.

\begin{tabular}{|c|c|c|}
\hline ALE 2 & ALE 1 & 1st Experiment \\
\hline $1.6 \times 10^{-6}$ & $2.1 \times 10^{-6}$ & a \\
\hline
\end{tabular}




\begin{tabular}{|c|c|c|}
\hline 169 & 225 & MSE \\
\hline \multicolumn{3}{|c|}{ Table (1): Results of experiment 1} \\
\hline ALE 2 & ALE 1 & 2nd Experiment \\
\hline $6.5 \times 10^{-7}$ & $1.9 \times 10^{-6}$ & 圆 \\
\hline 325 & 341 & MSE \\
\hline
\end{tabular}

Table (2): Results of experiment 2

\begin{tabular}{|c|c|}
\hline ALE is implemented using the 2D sign algorithm & Finally \\
\hline $1.5 \times 10^{-7}$ & M \\
\hline 192 & MSE \\
\hline
\end{tabular}

Table (3): Results of experiment 3
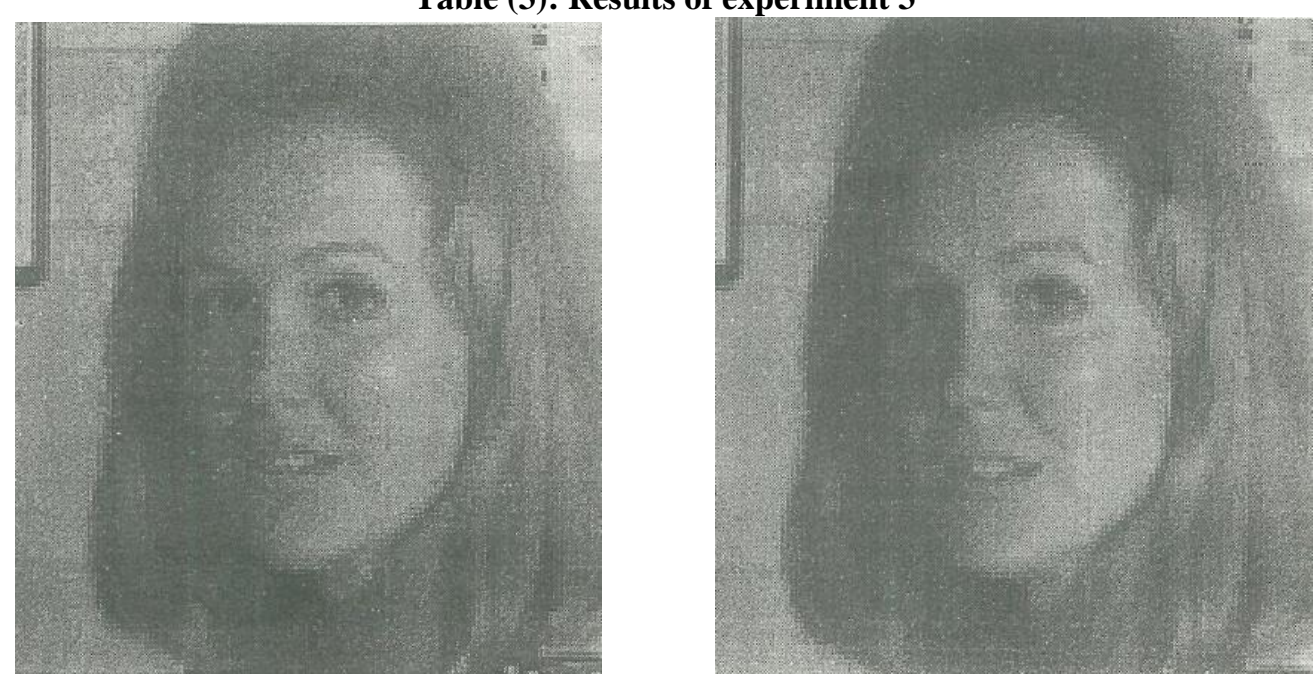

Figure (3): The original image of the girl

Figure (4): The image of the girl processed by ALE1 


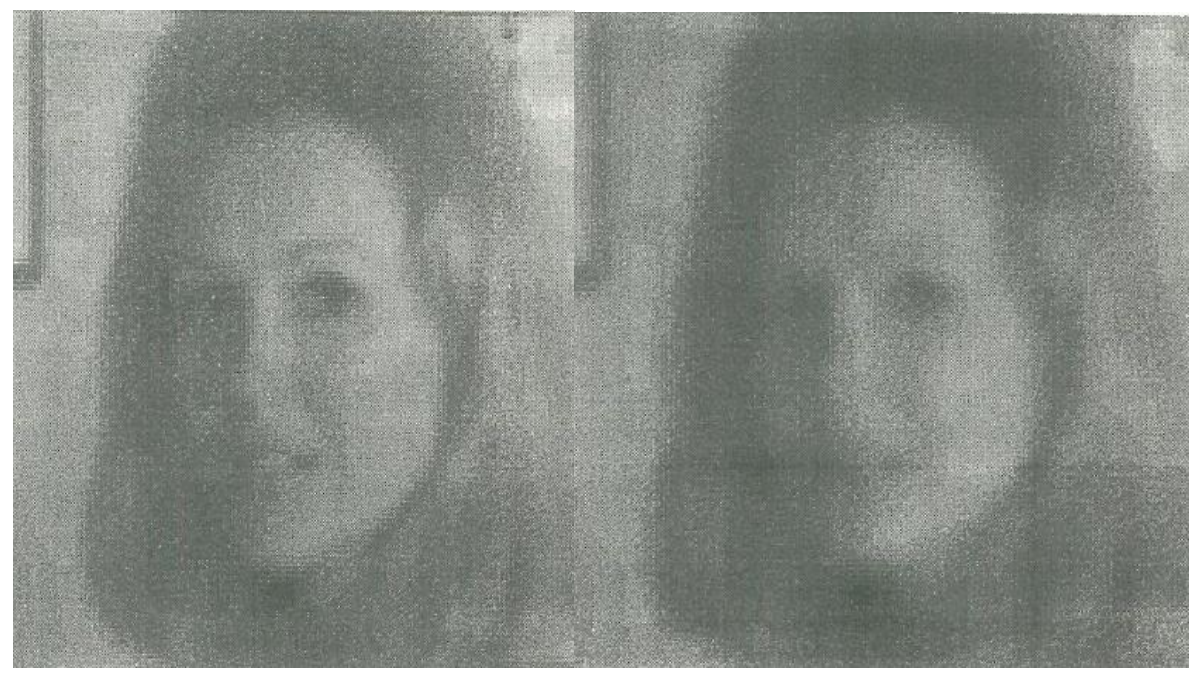

Fig. (5): The image of the girl processed by ALE1

Fig. (6): The noisy image $(\mathrm{SNR}=6 \mathrm{db})$

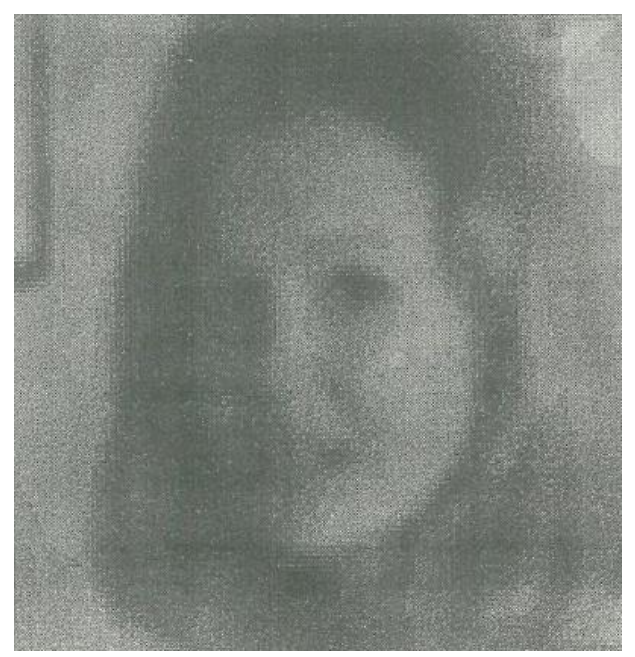

Fig. (7): The effect of noise reduction with ALE1

Fig. (8): The effect of noise reduction

\section{CONCLUSION}

with ALE2

The 2D adaptive filter is usually used when the statistical characteristics of the data processed do not match prior information on which the design of 2D adaptive recursive digital filters.

The modified model consists of two sections: the forward section and the backward. Each section is a conventional non-recursive adaptive filter. The stability of the filter is ensured due to the fact that the use of decision block bounds the feedback signal. The filter coefficients are adjusted in accordance with the 2D least mean square method (2D LMS) which converges to both 
the horizontal and the vertical directions of the 2D plane. Only some complexity is added when the quantizer is used in order to improve noise reduction.

Hence, the proposed filter does not impose degradation on the data being processed. To evaluate the performance of the proposed adaptive filter, it is implemented in the form of adaptive line enhancer (ALE). The ALE is capable of blurring images without imposing degradation. It also shows a good performance when it is used for noise reduction.

When comparing the ALE based on the proposed adaptive filter with that of Hadhoud. It proves to be superior. The ALE is implemented using the 2D sign algorithm, and compared with ALE adapted with the 2D LMS algorithm. When the control parameter $\mu$ is quite small both algorithm ensure the same MSE. The sign algorithm has the advantage of simple implementation. In a conclusion, the sign algorithm is recommended for the cases in which the main interest is the technical simplicity rather than convergence speed. 


\section{REFERENCES:}

[1] M. Hadhoud and D. Thomas, The two-Dimentional Adaptive LMS (TDLMS) Algorithm, IEEE Trans. Circuits Syst, Vol. CAS-35, No. 5, pp.485-494, May 1988.

[2] B. Widrow, et al. The complex LMS Algorithm, Proc. IEEE, Vol. 63, pp.719-725, April 1975.

[3] W. Jenkins and R. Fuast, A Constrained Two Dimensional Adaptive Filter with Reduced Computational Complexity, Proc. IEEE Int. Symp. Circuit Syst.,pp.2655-2658, 1988.

[4] T. Quatieri and E. Dudgean, Implementation of 2-D Digital Filters Iterative Methods, IEEE Trans. Acoustic Speech and Signal Processing, Vol. ASSP-30.pp.473-487, June 1982.

[5] C. Nikaias, A. Chryafis and A. Venetsanpoulos, The LU Decomposition Theorem and its Implications to the realization of 2-D Digital Filters. IEEE Trans. Acoustic Speech and Signal Processing, Vol. ASSP-33, No. 6, pp.694711, June 1985.

[6] M. Ohki and S. Hashiguchi, Two-Dimensional LMS Adaptive Filters, IEEE Trans. Con. Elect, Vol. 37, No. 1, pp.66-73, Feb 1991.

[7] S. Haykin, Introduction to Adaptive Filters. Macmillan Publishing Company, New York, 1983.

[8] J. Moscher, Adaptive Filter with Clipped Input Data Rept. 6796-1, Information Syst. Lap., Stanford Univ. June 1970.

[9] R. Gonzalez, Digital Image Processing. Paulwintz, 1987.

[10] A.F. Abdel-Razek, "Recursive 2-D Adaptive Digital Filter", $7^{\text {th }}$ International Conference on Electrical Engineering ICEENG 2010, MTC, 2427 May 2010, Military technical college, Cairo, Egypt.

[11] Takashi Hamanaka and Sadoc Maekana, "Structure of 2-D digital filters based on Marchesini Model", IEEE Trans. Speech and Signal Processing, Vol. ASSP-37, Feb. 2007.

[12] A. Vouofokis and G. Antonios, "Synthesis of 2-D digital filter with low sensitivity", IEEE Trans. Speech and Signal Processing, Vol. ASSP-39, Sept. 2012.

[13] Tim. I. Lookso, "Circuit and realization of 2-D all pass digital filter", IEEE Trans. Circuit \& System, Vol. 38, No. 9, Sept. 2011.

[14] T.Dabbous, "Identification for systems governed by nonlinear interval differential equations", Journal of industrial and management optimization, Vol. 8, No. 3, Aug. 2013. 